\title{
PRIMER REPORTE DE ACTINOMICOSIS PULMONAR EN LA PROVINCIA DE CIEGO DE AVILA - CUBA.
}

\author{
(First report of pulmonary actinomycosis in the Province of Ciego de Avila - Cuba)
}

\author{
Francisco Aparicio,A*., Miguel Suarez, $\mathrm{H}^{* *}$., \\ Elvio Luis Alvarez, $\mathbf{M}^{* *}$. \& Oreste Antune, $B^{* *}$. \\ *Hospital Provincial" Dr.Antonio Luaces Iraola" \\ **Centro Provincial de Higiene y Epidemiología, Ciego de Avila, Cuba.
}

Palabras clave: Actinomicosis pulmonar, Cuba.

Key Words: Pulmonary actinomycosis, Cuba.

\section{RESUMEN}

Se presenta el primer caso reportado de actinomycosis pulmonar en la provincia de Ciego de Avila (Cuba). El paciente presentó pérdida de peso, anorexia, y fiebre vespertina y en ocasiones, tos seca. En los exámenes, se observó una eritrosedimentación elevada, condensación de hase derecha a la radiografia de torax, opacidad total de los bronquios dellóbulo inferior derechoy parcialmente en los bronquios del lóbulo medio a la broncografia. Opacidad compatible con derrame interlohar. Se efectuó solo por biopsia el diagnóstico de actinomicosis pulmonar:

\section{INTRODUCCION}

La actinomicosis, es una infección común en el hombre y los animales, eatusala por varias especies de actinomicetos, siendo el principal agente etiológico $A c-$ tinomyces israelii (Actinomycotales, Actinomycetacoas). Se caracteriza por producir lesiones induradas, granulomatosas, de tipo supurativo, que dan lugar a la formación de fístulas (1, 10). Antiguamente los Actinomyces se consideraban relacionados con los hongos,pero en la actualidad se incluye en las Eubacterias anaerobias Gram(+) $(2,10)$.

Las principales localizaciones de sus cuadros clinicos son: región cervicofacial, torácica, abdominal y genital. La localización de estos microorganismos en los pulmones se caracteriza por su aspiración desde la cavidad oral, de los senos paranasales o por su inclusión en cuerpos exitraños $(3,11)$.

En general los pacientes pueden presentar tos, expectoración, astenia, pérdida de peso, disnea y dolor de

\section{SUMMARY}

This paper covers the first case of pulmonary actinomycosis reported in the province of Ciego de Avila (Cuha). The patient showed some loss in weight, anorexia,evening fever and occasionally a dy cough. Exams revealed a high eritrosedimentation, a right base condensation in the thorax radiography, a full opacity in bronchi from the right lower lobe and a partial one in hronchi from the middle lobe, as to the bronchography. The opacity was compatible with an interlobe overflow. The diagnosis of pulmonary actinomycosis was determined only by means of a biopsy.

tipo pleuritico. En cásos avanzados, puede haber fistulas a regiones vecinas y compromiso de las costillas. Radiologicamente, se han descrito imágenes de condensación a nivel de las bases y con menor frecuencia derrame asociado.

Como manifustaciones generales, se señala anemia, leucocitosis y eritrosedimentación elevada (4). El antimierobiano de elección es la penicilina (5), pero también se ha empleado la tctraciclina, eritromicina y clindamicina a altas dosis por tiempos prolongados, siendo necesario a veces el uso de la cirugia para el desbridamiento quirúrgico de los tejidos afectados (11).

Los 10 casos anteriores de actinomicosis diagnosticados en nuestro hospital no presentaron una localización pulmonar o torácica, es por ello que decidimos la presentación de este caso.

\section{PRESENTACION DEL CASO}

Paciente: M.B.G, sexo femenino, de 37 años, raza blanca, con antecedentes de hepatitis y sepsis urinaria. Un 
mes antes de su ingreso comienza a presentar dolor en la región posterior del hemitorax derecho que se acompaña de fiebre de $39^{\circ} \mathrm{C}$ y tos sin expectoración. Se trató con oxacilina obteniendose mejoría clínica pero no radiológica, por lo que es remitida a nuestro centro donde se decide su ingreso.

Datos positivos a la anamnesis. Tos seca, reliere padecer de anemia hace un año, de tipo ferripriva. Sepsis urinaria desde hace un año. Otros datos: pérdida de peso, anorexia y fiebre vespertina en ocasiones.

Datos positivos al examen físico. Paciente que deambula sin dificultad, marcha y facies no características de proceso patológico, en la piel, se observa a nivel de la región sacra paravertebral derecha, tres orilicios de fistulas sin supuración, ni fluctuación, ni cambios de coloración de la piel. Mucosas hipocoloreadas, cráneo y cara $\sin$ alteraciones, cuello y tiroides normales sin adenopatias, tórax sin alteraciones. Columna vertebral normal, abdomen normal y extremidades sin alteraciones.

Aparato respiratorio: expansibilidad normal, V.V., disminuidas en base derecha, submatidez en base derecha, murmullo vesicular abolido en base derecha.

Aparato cardiovascular: latido de la punta no visible ni palpable, ruidos taquicárdicos, R.R: 108 X', P.A: 110/ 70. Resto de los aparatos sin importancia.

\section{Análisis de Laboratorio:}
1. Hemoglobina: $8,8 \mathrm{~g} \%$
2. Hematocrito: $26 \mathrm{v} \%$
10. Glicemia: $109 \mathrm{mgs} \%$
3. Leucocitos: 7300
11. Urea: $24 \mathrm{mgs} \%$
12. Coprocultivo: (-)
4. Eosinofilo: 02
13. Sedimento de orina: (-)
5. Stabs: 02
6. Segmentados: 88
14. Hierro sérico: $52 \mathrm{mgs} \%$
15. Esputo BAAR: (-)

7. Linfocitos: 05

8. Monocitos: 03

9.Eritrosedimentación:

$144 \mathrm{~mm} / \mathrm{h}(1)$

$136 \mathrm{~mm} / \mathrm{h}(2)$

$115 \mathrm{~mm} / \mathrm{h}(3)$

\section{Estudios Radiológicos.}

Rayos X de Tórax: condensación de base derecha.Tomo grafia: en algunos cortes se observa borroso el hemidiafragma derecho, con ocupación del seno costo frénico. Broncografia: se opacilican totalmentelos bronquios del lóbulo inferior derecho y parcialmente los del lobulo medio. Persiste opacidad compatible con derrame interlobar.

Se realizó broncoscopia la cual es satisfactoria, en el curso de la misma, llama la atención un engrosamiento mucoso algo pálido a la entrada del bronquio tronco inferiro derecho.
Se tomaron muestras para biopsia, observandose en los cortes histológicos algunos gránulos de azufre pequeños que se tiñeron con Metamina argéntica y hematoxilinaeosina, presentando en sus bordes masas eosinófilas amorfas, elementos histicos y filamentos ramificados actinomicóticos.

Tratamiento. Inicialmente se recetó Penicilina cristalina endovenosa por 7 días y posteriormente Penicilina rapilenta por 10 días. Se continuó con Ampicilina por 4 semanas con buenos resultados.

\section{DISCUSION}

La actinomicosis torácica es una enfermedad rara, de origen endógeno, sin sintomas y signos característicos (inespecilicos); en aproximadamente el $90 \%$ de los casos se plantea otro diagnóstico, de éstos entre el 25 y $44 \%$ corresponden a una neoplasia y entre el 25 y $33 \%$ a otros trastomos pulmonares. En el $85 \%$ de los casos de las actinomicosis torácicas no son diagnosticadas con la toracotomía (6).

En afecciones pulmonares y pleurales de origen desconocido, se debe considerar la actinomicosis torácica en el diagnóstico diferencial (7).

Se expresa que esta afección ha cambiado considerablemente su manifestación clínica, siendo infrecuente ver los antiguos cuadros con destrucción de la pared del tórax $\mathrm{y}$ trayectos fistulosos de drenaje (8).

La enfermedad es más frecuente en adultos que en niños, y la confirmación de su diagnóstico en el laboratorio suele ser dilícil, debido al crecimiento lento de estos microorganismos (9).

\section{REFERENCIAS}

1. Donohue, D.E.; Brightman, A.II. (1995). Cervicofacial Actinomyces viscosus infection in a brazilian fila a case report and literature review. J. Am. Anim. Hosp. Assoc: 31: 501-505.

2. Fiorino, A.S. (1996). Intrauterine contraceptive device associated actinomycotic alscessand Actinomycesdetection on cervical smear. Obstet Gynecol. 87: $142-149$

3.Rodriguez, P.; Sandoval, R.; Rodriguez, J.C. (1990). Actinomicosis torácica. Enfermedades respir. cir. torác. 6:204-208

4. Coclho,F. \& Jobo, C.(1990).Pulmonary cavities colonized by Actinomycetes. Report of 6 cases. Rev. Inst. Med. Trop. Sao Paulo 32:6366

5. Viotti, J. A.; Jaremciuk, N. S.; Lodolo, A. \& Giraudo, R. (1991). Actinomicosisde pared aldominal. Presentaciónde un caso. Rev. Fac. Cienc. Med. Córdoba. 49:35-38

6. Nohr,B.1); Schmitt, B.; Goller; T. \& Lenz, J. (1995). Thoracic actinomy'cosis. Chirurg. 66:81 8-822 
7. Orth,M.; Achatzy,R.; Macha,M.M.; Müller,K.M.; Wiebe,V. \& Schultze-Weminghaus, G. (1995). Pleuromediastinitis in pulmonary actinomycosis as a rare differential bronchial carcinoma diagnosis. Pneumologie. 49:505-508

8. Cabezas,S.; Francisco,I.; Cortes, L.; Weitz,B.; Cancino,A.: Dalbora, P.; Avila, P.; Vallejo,A. \& Johow, P.(1992). Actinomicosis pulmonar. Rev. Chil. Cir. 44:343-346

9. Hoffmeister, R.; Rios,C.; Contreras,G.; Jadue, J.; Joyas,M. \& Alejandro,M. (1989). Actinomicosis torácica. Rev.Chil.Pediatr. 60:173178
10. Schaal, K (1986). Genus Actinomyces. In: Bergey's Manual of Sistematic Bacteriology. WilliamsWilkins, Baltimore. pp. 1383-1418

11. Casal,M. \& Rodriguez,F.C.(1996).Actinomyces, Nocardia y Mycobacterium. En:García-Rodríguez, J.A. \& Picazo, J.J. (Eds.) Microbiología Médica. 1. Microbiología Médica General. Mosby/Doyma Libros, S.A. pp. 358-362 